\title{
Multiple microRNAs as biomarkers for early breast cancer diagnosis
}

\author{
JI YOUNG JANG ${ }^{1 *}$, YEON SOO KIM ${ }^{2 *}$, KYUNG NAM KANG ${ }^{1}$, \\ KYO HYUN KIM ${ }^{2}$, YU JIN PARK ${ }^{1}$ and CHUL WOO KIM ${ }^{1}$ \\ ${ }^{1}$ BIOINFRA Life Science Inc., Jongno-gu, Seoul 03127; ${ }^{2}$ DIOGENE Inc., \\ Bundang-gu, Seongnam-Si 13486, Republic of Korea
}

Received June 5, 2020; Accepted December 7, 2020

DOI: $10.3892 / \mathrm{mco} .2020 .2193$

\begin{abstract}
MicroRNA (miRNA or miR) is stably present in plasma. It has been reported that miRNA could be used for detecting cancer. Circulating miRNAs are being increasingly recognized as powerful biomarkers in a number of different pathologies, including in breast cancer. The aim of the current study was to establish and validate miRNA sets that are useful for the early diagnosis of breast cancer. Specifically, the current study intended to determine whether miRNA biomarkers were tumor-specific and to statistically verify whether circulating miRNA analysis could be used for breast cancer diagnosis. In the present study, a total of nine candidate miRNA biomarkers were selected by examining reference miRNAs associated with the generation and progression of breast cancer to identify novel miRNAs that could be used to detect early breast cancer. A total of 226 plasma samples from patients with breast cancer were used. In addition, 146 plasma healthy samples were used as non-cancer controls. These samples were divided into training and validation cohorts. The training cohort was used to identify a combination of miRNA that could detect breast cancer. The validation cohort was used to validate this combination of miRNA. Total RNAs were isolated from collected samples. A total of 9 miRNAs were quantified using reverse-transcription quantitative PCR. A total of nine candidate miRNA expression levels were compared between patients with breast cancer and healthy controls. It was indicated that combinations of two or more of the nine miRNAs could detect breast cancer with higher accuracy than the use of a single
\end{abstract}

Correspondence to: Dr Chul Woo Kim, BIOINFRA Life Science Inc., Daehak-ro 49, Jongno-gu, Seoul 03127, Republic of Korea

E-mail: chulwoo.kim@bioinfra.co.kr

*Contributed equally

Abbreviations: pri-miRNAs, primary miRNAs; pre-miRNA, precursor miRNA

Key words: exosome, pre-microRNA, breast cancer, liquid biopsy, early diagnosis biomarker. As a representative example, combinations of four miRNAs (miR-1246+miR-206+miR-24+miR-373) of the nine miRNAs had a sensitivity of $98 \%$, a specificity of $96 \%$ and an accuracy of $97 \%$ for breast cancer detection in the validation cohort. The results of the present study suggest that multiple miRNAs could be used as potential biomarkers for early diagnosis of breast cancer. These biomarkers are expected to overcome limitations of mammography when used as an auxiliary diagnosis of mammography.

\section{Introduction}

Breast cancer accounts for $25.2 \%$ of all female cancers worldwide. It has the highest incidence among female cancers (1), displaying a rapidly increasing trend. Among imaging medical diagnostic methods used for early diagnosis of breast cancer, mammography is the only clinically proven test method (2). However, mammography has problems such as false negative diagnosis and excessive additional examination according to false positive diagnosis, unnecessary biopsy, psychological burden and radiation exposure. The sensitivity of mammography ranges from 62.2 to $89.5 \%$ with a specificity of $62.7 \%$. More women have dense breasts in Korea than in western countries. The sensitivity of mammography is significantly lower for dense breasts. Thus, the need for auxiliary test is being emphasized (3). Ultrasonography of the breast is mainly used as an auxiliary test for mammography. However, breast ultrasound used as an auxiliary test has the following problems. First, it has a high dependence on test equipment and testers. Second, scientific and objective evidence for the mortality rate from breast cancer has not been established yet. Third, false positives cannot be avoided, and additional tests and biopsy tests are required. Fourth, early diagnosis of breast cancer is difficult due to calcified lesions (4). Therefore, it is necessary to develop a new and auxiliary test for diagnosing breast cancer in order to solve these limitations of mammography.

Invasive biopsy has disadvantages in that the patient suffers severely. It also has side effects due to infection that might require a recovery period after hospitalization and examination. On the other hand, a non-invasive method using blood (blood collection) has the advantages of being very simple and painless. It requires no recovery period after hospitalization and examination. In addition, such liquid biopsy 
has the advantage of avoiding side effects of tissue biopsy. Early diagnosis is possible even for potential patients who have not developed cancer. It is advantageous to periodically observe the progress of treatment of patients who have already developed the disease (5-7). Therefore, screening high-risk groups of patients for breast cancer using liquid biopsy and the development of early diagnosis methods can compensate for problems of existing tissue biopsy and greatly contribute to the reduction of medical expenses.

Exosome is a group of small membranous vesicles that are shed into body fluids or extracellular environment by tumoral or nontumoral cells. These vesicles play pivotal roles in cellular communication through shuttling between donor and recipient cells (8). The lumen of an exosome has different components such as DNA, RNA, lipids and proteins, representing bioactive molecules in donor cells. miRNAs are among cargos of exosomes. They are involved in different processes such as angiogenesis and metastasis of cancer (9). Exosomes are nano-vesicles present in the circulation. They are involved in cell-to-cell communication and the regulation of different biological processes. miRNAs as cargos of exosomes are potential biomarkers (10). Due to interesting features of exosomal miRNAs, they can be promising biomarkers for cancer diagnosis (11). Due to the presence of exosomes in various body fluids and the stability of miRNAs in exosomes, exosomal miRNAs might be a new class of biomarkers for early and minimally invasive cancer diagnosis (12).

Recently, the emergence of miRNA, a small non-proteincoding RNA that plays an important role in tumor initiation and progression, has opened up new opportunities for early cancer diagnosis $(13,14)$. miRNAs are 19-25 nucleotides regulatory non-coding RNA molecules that regulate expression levels of a wide variety of genes by sequence-specific base pairing for 39 untranslated regions of target mRNA, resulting in mRNA degradation or inhibition of translation. Evidence suggests that miRNA expression profiles can cluster similar tumor types together more accurately than expression profiles of protein-coding mRNA genes (15). Furthermore, miRNA expression signatures have been used to predict prognosis $(16,17)$. As a screening tool that is easily accepted by the general population, it would be desirable to detect cancer accurately, without resorting to an invasive procedure. Recently, several reports have suggested that circulating miRNAs are stable and detectable in serum/plasma and that levels of some miRNAs in breast cancer patients are specifically elevated $(18,19)$.

Canonically, biogenesis of miRNAs starts in cell nucleus where DNA containing miRNAs is transcribed by RNA polymerase II to generate primary miRNAs (pri-miRNAs) (20). These pri-miRNAs are then processed by a microprocessor complex consisting of RNase type III endonuclease Drosha and an essential cofactor (DiGeorge syndrome critical region 8)/Pasha (protein containing two double-stranded RNA binding domains) to generate precursor miRNA (pre-miRNAs) (21,22). Pre-miRNAs are then exported to the cytoplasm by an exchange factor of guanine Ran nucleotide (GTP-binding nuclear protein Ran) and an exportine-5 receptor. They are then processed by another RNase type III endonuclease known as Dicer, releasing 22-nucleotide miRNA duplex. One strand of the RNA duplex is selected to be subsequently loaded into the RNA-induced silencing complex (RISC), along with argonaut (AGO2) and GW182 $(23,24)$. Whether incorporation of miRNA into exosomes occurs at pre-miRNA or mature miRNA level remains unclear. However, it has been reported that precursor miRNA contains a higher ratio of mature miRNA (25). Based on years of research experience comparing mature miRNA and pre-miRNA expression in the same sample from our team (data not shown), the aim of this study was to analyse pre-miRNA expression, rather than mature miRNA, as a biomarker for early diagnosis of breast cancer.

To obtain accurate results in real-time PCR, it is important to accurately combine templates and primers. Dumbbell-like structural primer for pre-miRNA amplification is our team's original technique that can minimise real-time PCR nonspecific reactions (26). Thus, it was used in the present study. In this study, we tried to construct a set of multiple pre-miRNA biomarkers that are optimal for developing new blood-based early diagnostic assays for breast cancer.

\section{Materials and methods}

Cohorts and plasma samples. In this study, 226 breast cancer patients and 146 healthy control plasma were used. Specifically, 146 breast cancer patients and 90 healthy control serum were used in the initial discovery study. Then 80 breast cancer patients and 56 healthy control plasma were used to validate the classification model of this study. Plasma samples from breast cancer patients with cancers were obtained from Korea Regional Biobank of Busan National University Hospital, Inje University Busan Paik Hospital and Chonnam National University Hwasun Hospital. These breast cancer samples were obtained before any therapeutic approaches were performed. This study was ethically approved by our Institutional review board from the IRB (BIOINFRA Life Science Institutional Review Board). The member was of this review board Jung Bo Kyung, Kim Chul Woo, Shin Yong Sung, and Kim Hee Yoon. Samples were stored at $-80^{\circ} \mathrm{C}$ until analysed. Plasma samples from asymptomatic healthy donors were obtained from Korea Regional Biobank of Ajou University Hospital, Wonkwang University Hospital, Jeonbuk National University Hospital, Chonnam National University Hospital and Kyungpook National University Hospital. Healthy controls with a known history of cancer, high-grade dysplasia, autoimmune disease, chronic kidney disease, pregnancy, or inflammatory conditions that needed medical management were excluded. The clinical stage of cancer was determined by the final pathological diagnosis after resection according to the 7 th edition of the Union for International Cancer Control tumor-node-metastasis classification.

Isolation of RNA from plasma. Total RNAs were extracted from plasma samples (300 $\mu \mathrm{l})$ using a nucleic acid automatic extraction equipment (Smart Lab Assist-24, Korea KETT) and finally eluted with $150 \mu \mathrm{l}$ RNase-free water. Concentration of extracted total RNAs were measured. Measured concentrations were analysed to correct concentration values of all samples.

Removal of genomic DNA and cDNA synthesis by reverse transcription $(R T)$. The gDNA was removed from the extracted 
total RNA. Total RNA was then used to synthesise cDNA which was then quantified using an internal control primer. gDNA removal and RT were performed using PrimeScript ${ }^{\mathrm{TM}}$ RT reagent kit with gDNA Eraser (product code RR047A, Takara).

Analysis of miRNA gene expression by quantitative real time polymerase chain reaction $(q P C R)$. In the first multiplex PCR, a total of nine miRNA primers $(2.5-30 \mathrm{pmol})$ $(1 \mu \mathrm{l})$ were mixed with $4 \mu \mathrm{l}$ of template cDNA and $5 \mu \mathrm{l}$ of 2X multiplex PCR master mix. Primer concentrations for each miRNA for secondary real-time PCR were in the range 4-10 pmol. The primary PCR product was diluted 1:10 and used for secondary real-time PCR analysis. One $\mu 1$ of each primer, $4 \mu \mathrm{l}$ of the primary PCR product and $5 \mu \mathrm{l}$ of $2 \mathrm{X}$ SYBR master mix were mixed to make a final volume of $10 \mu \mathrm{l}$ for PCR. Primer sequences used for PCR are shown below. $\mathrm{X}$ in primer sequence is inosine. miR-223 (NR_029637.1) forward primer 5'-GACCAXXXXXAGTTGGACACTCCA TGTGGTC-3' and reverse primer 5'-AGTGCXXXXXTG GTAAGCATGTGCCGCACT-3'; miR-1246 (NR_031648.1) forward primer 5'-CAGGTXXXXXTGGAGCAGGAGTGG ACACCTG-3' and reverse primer 5'-CAATCXXXXXAT TGCTAGCCTATGGATTG-3'; miR-206 (NR_029713.1) forward primer 5'-AGCATXXXXXTGCTTCCCGAGGCC ACATGCT-3' and reverse primer 5'-AAGTGXXXXXACTTG CCGAAACCACACACTT-3'; miR-24 (NR_029497.1) forward primer 5'-CTGTGXXXXXGTGCCTACTGAGCTGAA ACACAG-3' and reverse primer 5'-CACTGXXXXXGTTCC TGCTGAACTGAGCCAGTG-3'; miR-373 (NR_029866.1) forward primer 5'-CAGACXXXXXCGCTTTCCTTTTTG TCTG-3' and reverse primer 5'-GTGCTXXXXXGACAC CCCAAA ATCGAAGCAC-3'; miR-21 (NR_029493.1) forward primer 5'-CAGTCXXXXXGTCGGGTAGCTTAT CAGACTG-3' and reverse primer 5'-CAGTCXXXXXCAGAC AGCCCATCGACTG-3'; miR-6875 (NR_106935.1) forward primer 5'-CTTCTXXXXXGACCCAGGACAGGAGAA G-3' and reverse primer 5'-GTGATXXXXXGCAGGAAG AATGCAAATCAC-3'; miR-202 (NR_030170.1) forward primer 5'-GGCCAXXXXXGCATATACTTCTTTGAGGAT CTGGCC-3' and reverse primer 5'-CATGGXXXXXGA CCGCCCCGTTTTCCCATG-3'; miR-219B (NR_039815.1) forward primer 5'-ACATCXXXXXGGAGCTCAGCCACA GATGT-3' and reverse primer 5'-GTTTGXXXXXGCGCC ACTGATTGTCCAAAC-3'.

Statistical methods. Mann-Whitney U test (Wilcoxon rank-sum test) was used to determine whether these nine candidate microRNA markers might be significantly different between breast cancer patients and healthy controls. $\mathrm{P}<0.01$ was considered to indicate a statistically significant difference. Spearman correlation test between markers was also performed to evaluate the independence of candidate markers. Next, to minimise the influence of outliers in biomarker measurement values, a $\log _{10}$ transformation was performed for measurement values. This study included 226 total breast cancer samples and 146 healthy control samples. To select an optimal marker panel, a training data set (146 breast cancer and 90 healthy controls) and a validation data set ( 80 breast cancer and 56 healthy controls) were used. To select the optimal marker panel, 511 biomarker panel sets were generated. This number was the number of all possible combinations for these nine candidate biomarkers. For the training data set, a classification model corresponding to 511 biomarker panel sets was generated using Random Forest (RF) algorithm, one non-linear classification technique. The classification model was then verified using the validation set. Criteria for selecting the optimal marker panel should be excellent in performance such that the area-under-the-curve (AUC) of the receiver operation characteristic (ROC) calculated at model generation is close to 1 . It was confirmed that the performance was similar for the validation set. All analysis procedures were performed using R statistical package version 3.5.1 (https://www.r-project.org/ and https:/ftp.harukasan.org/CRAN/index.html), a statistical analysis tool.

\section{Results}

Performance value of each candidate miRNA biomarkers in the training set. As summarised in Table I, nine candidate miRNAs were selected based on reference search (27-40). First, expression levels of these nine candidate miRNAs (miR-223, 1246, 206, 24, 373, 21, 6875, 202 and 219B) predicted to be important for screening normal and breast cancer patients were analysed. Samples used in the analysis included 146 healthy controls (31 from the Korea Regional Biobank of Ajou University Hospital, 25 from Wonkwang University Hospital, 16 from Jeonbuk National University Hospital, 23 from Chungnam National University Hospital, 27 from Kyungpook National University Hospital and 24 from Ajou University Hospital), together with 226 breast cancer patients (147 from the Korea Regional Biobank of Busan National University Hospital, 39 from Inje University Busan Paik Hospital and 40 from Chonnam National University Hwasun Hospital). At this time, all normal plasma samples were female samples. The expression level of each miRNA was a cross point between a miRNA amplification curve and a threshold line in a threshold cycle $(\mathrm{Ct})$, meaning a relative measurement of the target miRNA concentration in a real-time PCR reaction. All nine candidate miRNAs were found to be meaningful for distinguishing between normal and breast cancer patients (Table II). This was also confirmed in a bar graph boxplot (Fig. 1).

Correlation analysis for nine miRNA biomarkers. Results of correlation analysis for these nine miRNA biomarkers (miR-223, 1246, 206, 24, 373, 21, 6875, 202 and 219B) are shown in Table III. Correlations among nine miRNA biomarkers were analysed using Spearman's correlation analysis. The degree of correlation was generally expressed as follows: Correlation coefficient $\mathrm{R}=1$, same; $\mathrm{R} \geq 0.9$, very high correlation; $0.7 \leq \mathrm{R}<0.9$, high correlation; $0.4 \leq \mathrm{R}<0.7$, slightly higher correlation; $0.2 \leq \mathrm{R}<0.4$, low correlation; and $\mathrm{R} \leq 0.2$, little correlation. No miRNA had a very high or high correlation. It was confirmed that correlations of miR-223 with miR-373 and miR-202 were slightly higher. On the other hand, miR-373 had a somewhat higher correlation with miR-223 and miR-206. These results show that it is possible to select miRNAs that are not highly correlated when selecting an optimal biomarker combination. However, when each miRNA plays an important function in breast cancer onset and 
Table I. Preclinical study report of nine candidate miRNAs.

\begin{tabular}{|c|c|c|c|}
\hline Reference name and year & Biomarker & Function & Refs. \\
\hline $\begin{array}{l}\text { Pinatel et al 2014; } \\
\text { Yoshikawa et al } 2018\end{array}$ & $\operatorname{miR}-223$ & $\begin{array}{l}\text { miR-223 is a coordinator of breast cancer progression; The } \\
\text { expression level is higher in the patients IDC than with DCIS }\end{array}$ & $(27,28)$ \\
\hline $\begin{array}{l}\text { Fu et al } 2016 \\
\text { Li et al } 2017\end{array}$ & $\operatorname{miR}-1246$ & $\begin{array}{l}\text { miR-382-3p, }-598-3 p,-1246 \text {, and - } 184 \text { are all involved in the development } \\
\text { of breast cancer, and are promising biomarkers for breast cancer detection; } \\
\text { Exosomal microRNA miR-1246 promotes cell proliferation, invasion and } \\
\text { drug resistance by targeting CCNG } 2 \text { in breast cancer }\end{array}$ & $(29,30)$ \\
\hline Zhou et al 2019 & miR-206 & $\begin{array}{l}\text { miR-206 promotes cancer progression by targeting full-length } \\
\text { Neurokinin- } 1 \text { receptor in breast cancer }\end{array}$ & $(31)$ \\
\hline $\begin{array}{l}\text { Khodadadi-Jamayran } \\
\text { et al } 2018 \\
\text { Lu et al } 2015\end{array}$ & $\operatorname{miR}-24$ & $\begin{array}{l}\text { Prognostic role of elevated mir-24-3p in breast cancer and its association } \\
\text { with the metastatic process; miRNA-24-3p promotes cell proliferation and } \\
\text { inhibits apoptosis in human breast cancer by targeting p } 27 \mathrm{Kip} 1\end{array}$ & $(32,33)$ \\
\hline $\begin{array}{l}\text { Eichelser et al 2013; } \\
\text { Piasecka et al } 2018\end{array}$ & $\operatorname{miR}-373$ & $\begin{array}{l}\text { miR-373 is known to be relevant for cancer development, progression, } \\
\text { and metastasis; mR-373 is associated with EMT/CSC and invasion }\end{array}$ & $(34,35)$ \\
\hline $\begin{array}{l}\text { Asaga et al 2011; } \\
\text { Corcoran et al } 2011\end{array}$ & $\operatorname{miR}-21$ & $\begin{array}{l}\text { Circulating miR-21 has diagnostic and prognostic potential in breast } \\
\text { cancer }\end{array}$ & $(36,37)$ \\
\hline Shimomura et al 2016 & $\operatorname{miR}-6875$ & $\begin{array}{l}\text { A combination of miR-1246, miR-1307-3p, miR-4634, miR-6861-5p, and } \\
\text { miR- } 6875-5 p \text { measured from serum can be used to detect breast cancer in } \\
\text { the early stages, and to differentiate breast cancer from pancreas/biliary } \\
\text { tract/prostate benign diseases or other cancers }\end{array}$ & $(38)$ \\
\hline Schrauder et al 2017 & miR-202 & $\begin{array}{l}\text { miR-202 was significantly upregulated in whole blood samples of } \\
\text { early-stage breast cancer patients }\end{array}$ & $(39)$ \\
\hline Zhao et al 2017 & $\operatorname{miR}-219 B$ & $\begin{array}{l}\text { Gga-miR-219b targeting BCL11B suppresses proliferation, migration, } \\
\text { and invasion of Marek's disease tumor cell MSB1 }\end{array}$ & $(40)$ \\
\hline
\end{tabular}

miR, microRNA; IDC, invasive ductal carcinoma; DCIS, ductal carcinoma in situ; CCNG2, Cyclin-G2; EMT, epithelial-to-mesenchymal transition; Gga, Gallus gallus; BCL11B, B-cell chronic lymphocytic/lymphoma 11B; MSB1, MDV-transformed lymphoid cell line.

Table II. Result of the U-test analysis of single miRNA biomarkers.

\begin{tabular}{lll}
\hline Rank & miRNA biomarker & P-value \\
\hline 1 & miR-223 & $1.28 \times 10^{-53}$ \\
2 & miR-1246 & $1.47 \times 10^{-53}$ \\
3 & miR-206 & $1.17 \times 10^{-49}$ \\
4 & miR-24 & $1.22 \times 10^{-49}$ \\
5 & miR-373 & $1.58 \times 10^{-49}$ \\
6 & miR-21 & $1.53 \times 10^{-46}$ \\
7 & miR-6875 & $2.24 \times 10^{-43}$ \\
8 & miR-202 & $1.73 \times 10^{-39}$ \\
9 & miR-219B & $4.99 \times 10^{-23}$ \\
\hline
\end{tabular}

miR, microRNA.

progression, these miRNAs should be included in the combination set, even if there is a rather high correlation.

Combination of multiple miRNA biomarkers for breast cancer diagnosis. To create a classification model, total samples were divided into samples for model generation (training set) and samples for model verification (validation set). Data for model generation and verification were distributed at a ratio of $\sim 2: 1$ (training: Validation set) (Fig. 2 and Table IV). Samples for model generation and verification were randomised. At this time, age information was not reflected. Table IV shows the accuracy of a single miRNA in training and validation sets. Table $\mathrm{V}$ shows examples of a representative set of multiple miRNA biomarkers that meet all rules. Tables IV and V confirmed that the performance of multiple biomarkers was improved compared to that of a single biomarker. In addition to examples given in Tables V and SI-III of Supplementary Information (SI) shows examples of two to four combinations of miRNA biomarkers out of 511 biomarker panel sets that met all rules. Results showed that multiple markers had higher accuracy for early diagnosis of breast cancer than single markers.

\section{Discussion}

To improve the performance of breast cancer treatment, discovery and accurate diagnosis are very important. Representative imaging methods used for breast examination are mammography, breast ultrasound and breast magnetic 
Table III. Analysis of correlation between the nine miRNAs.

\begin{tabular}{lccccccccc}
\hline Correlation & miR-223 & miR-1246 & miR-206 & miR-24 & miR-373 & miR-21 & miR-6875 & miR-202 & miR-219B \\
\hline miR-223 & 1.00 & 0.28 & 0.45 & 0.12 & 0.60 & 0.27 & 0.38 & 0.59 \\
miR-1246 & 0.28 & 1.00 & 0.14 & 0.29 & 0.38 & 0.36 & 0.19 & 0.43 \\
miR-206 & 0.45 & 0.14 & 1.00 & 0.34 & 0.56 & 0.17 & 0.41 & 0.23 \\
miR-24 & 0.12 & 0.29 & 0.34 & 1.00 & 0.27 & 0.14 & 0.28 & 0.16 \\
miR-373 & 0.60 & 0.38 & 0.56 & 0.27 & 1.00 & 0.32 & 0.41 & 0.51 \\
miR-21 & 0.27 & 0.36 & 0.17 & 0.14 & 0.32 & 1.00 & 0.17 & 0.36 \\
miR-6875 & 0.38 & 0.19 & 0.41 & 0.28 & 0.41 & 0.17 & 1.00 & 0.20 & 0.12 \\
miR-202 & 0.59 & 0.43 & 0.23 & 0.16 & 0.51 & 0.36 & 0.20 & 1.00 & 0.15 \\
miR-219B & 0.13 & 0.13 & 0.07 & 0.00 & 0.12 & 0.34 & 0.15 & 0.01 & 0.01 \\
\end{tabular}

miR, microRNA; $R=1$, same; $R \geq 0.9$, very high correlation; $0.7 \leq \mathrm{R}<0.9$, high correlation; $0.4 \leq \mathrm{R}<0.7$, slightly higher correlation; $0.2 \leq \mathrm{R}<0.4$, low correlation; and $\mathrm{R} \leq 0.2$, little correlation.

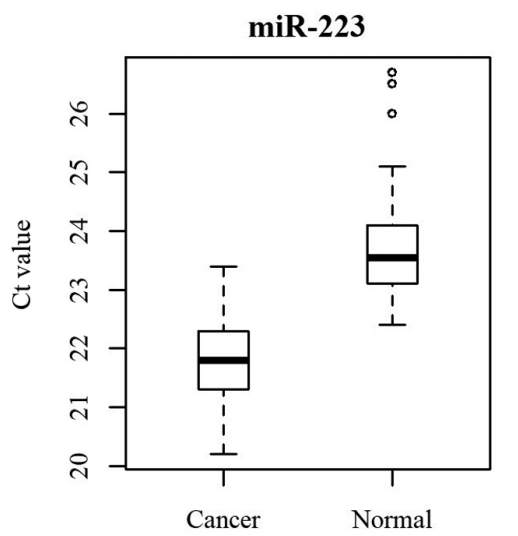

miR-24

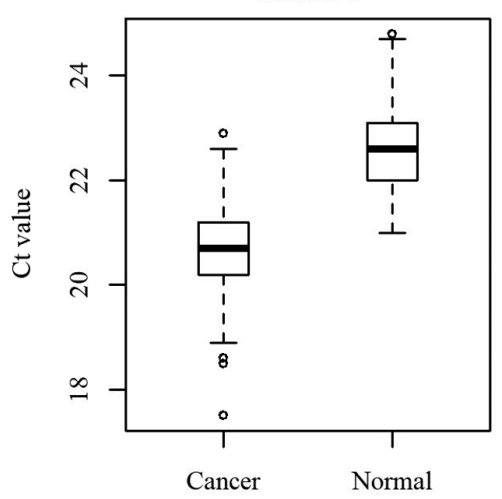

miR-6875

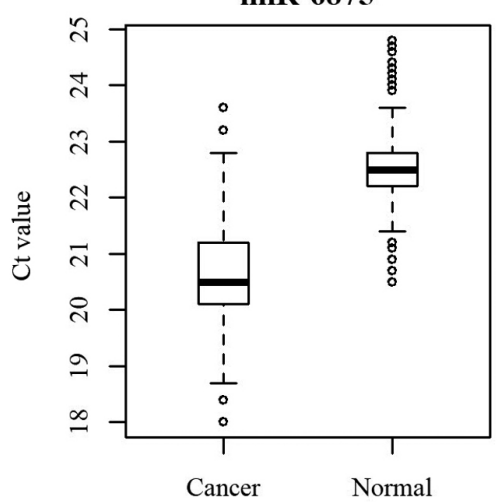

miR-1246

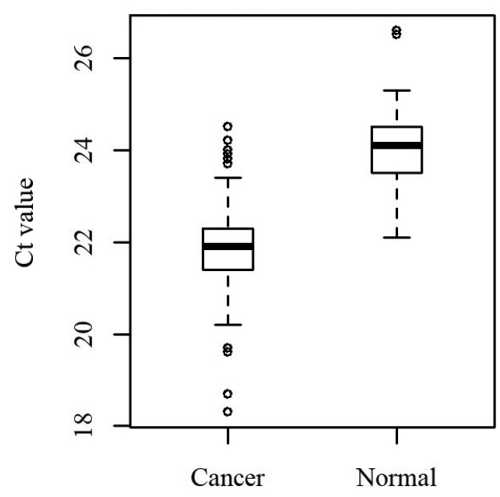

miR-373

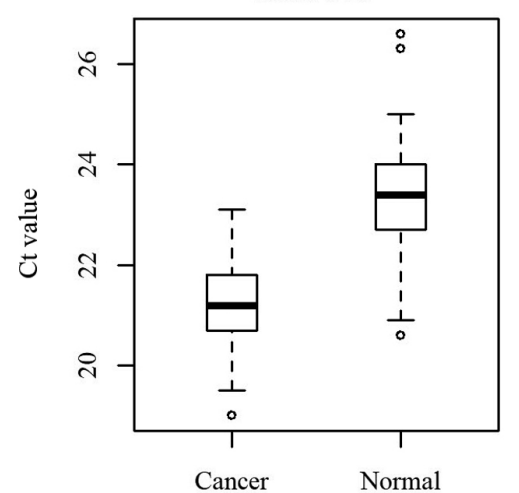

miR-202

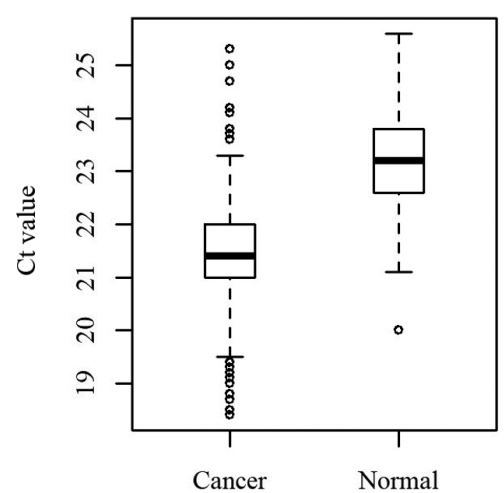

miR-206

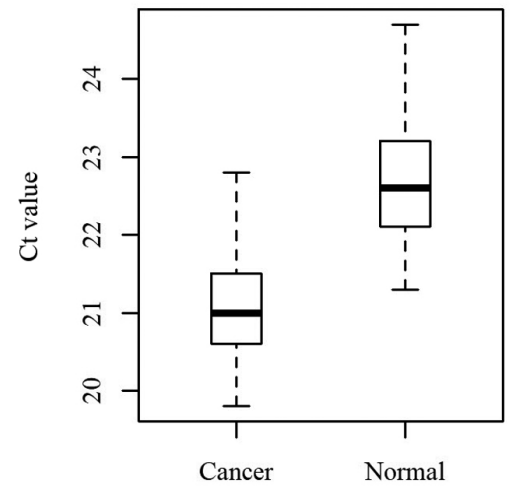

miR-21

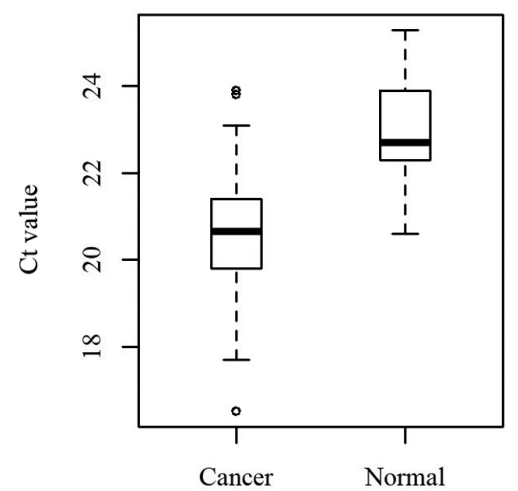

miR-219B

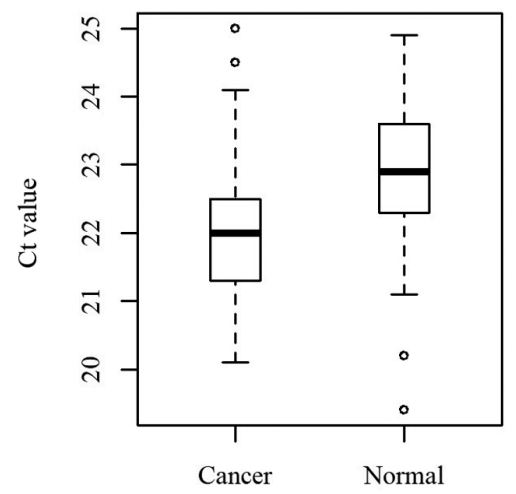

Figure 1. Bar graph of U-test analysis of single miRNA biomarkers. All nine miRNAs were indicated to be meaningful for distinguishing between healthy patients and patients with breast cancer in the bar graph. miRNA or miR, microRNA. 


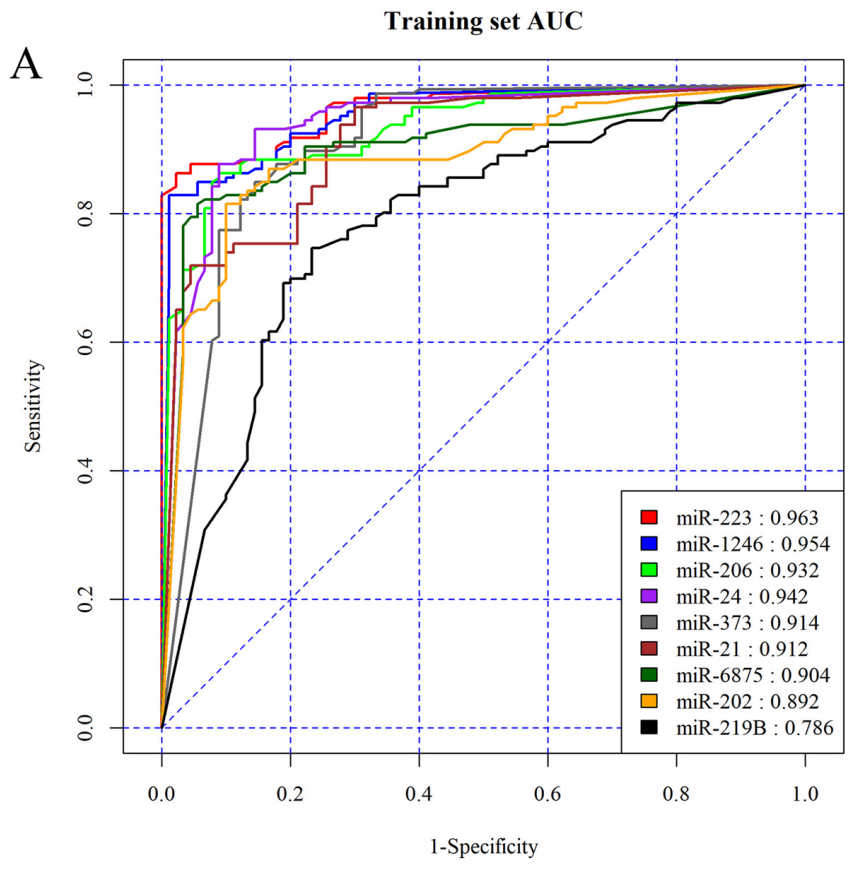

$\mathrm{B}$

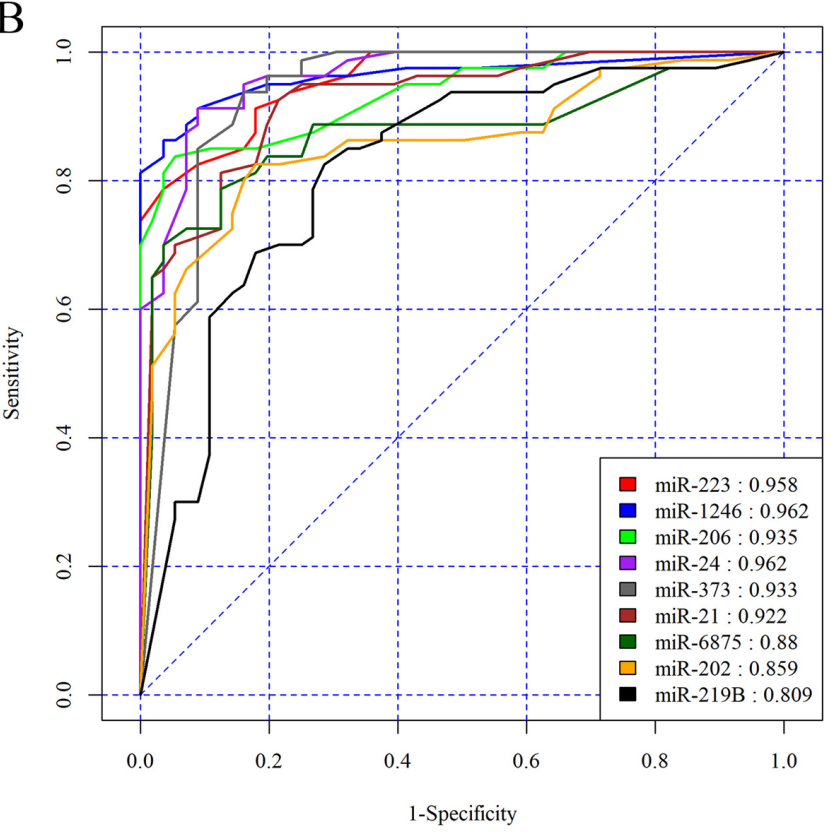

Figure 2. ROC curves of training and validation sets for a single biomarker of breast cancer. To create a classification model, total samples were divided into samples for model generation (training set) and samples for mode verification (validation set). Data for model generation and verification were distributed at a ratio of $\sim 2: 1$ (training: Validation set). Samples for mode generation and verification were randomized. At this time, age information was not reflected. The $\mathrm{x}$-axis represents specificity while the $\mathrm{y}$-axis represents sensitivity in the (A) training set and (B) validation set ROC curve. miRNA or miR, microRNA; AUC, area under the curve; ROC, receiver operator characteristic.

resonance imaging (MRI). Mammography is the effective diagnostic tool and screening test for breast cancer and has been scientifically proven to be able to lower breast cancer and mortality. However, mammography can lead to false negative diagnosis and false positive diagnosis, resulting in excessive additional examination and unnecessary biopsy with disadvantages such as inpatient treatment, psychological burden and
Table IV. AUC values of a single biomarker of breast cancer using statistical methods in the training and validation sets.

\begin{tabular}{llcc}
\hline Index & Biomarker & $\begin{array}{c}\text { Training } \\
\text { set AUC }\end{array}$ & $\begin{array}{c}\text { Validation } \\
\text { set AUC }\end{array}$ \\
\hline 1 & miR-223 & 0.963 & 0.958 \\
2 & miR-1246 & 0.954 & 0.962 \\
3 & miR-206 & 0.932 & 0.935 \\
4 & miR-24 & 0.942 & 0.962 \\
5 & miR-373 & 0.914 & 0.933 \\
6 & miR-21 & 0.912 & 0.922 \\
7 & miR-6875 & 0.904 & 0.880 \\
8 & miR-202 & 0.892 & 0.859 \\
9 & miR-219B & 0.786 & 0.809 \\
\hline
\end{tabular}

AUC, area under the curve.

radiation exposure. Moreover, sensitivity of mammography is lower for young women and women with dense breasts. Assisted mammography methods include breast ultrasound and magnetic resonance imaging. However, these methods also have limitations (41-43). For this reason, many attempts have been made recently to select high-risk patients for breast cancer. Liquid biopsy has been used as an aid to early diagnosis of breast cancer. A liquid biopsy has the advantage of being non-invasive for early detection of cancer. It also enables multiple repetitions and easy monitoring of the disease (44).

Several previous studies have demonstrated the value of circulating miRNAs in breast cancer diagnosis. A number of new breast cancer related miRNAs have been identified $(45,46)$. However, there have not been comprehensive reports of numerous miRNAs using plasma samples of breast cancer patients. In this study, we analysed expression levels of a number of plasma miRNAs expected to be valuable for early diagnosis of breast cancer in an attempt to obtain an optimal combination of multiple miRNAs. Dumbbell-like structural primer for pre-miRNA amplification by real-time PCR as our team's proprietary technology that could minimise non-specific PCR in real time was used in the present study.

Results of this study showed AUC values of 0.809-0.962 for the classification model with a single miRNA alone (Table IV). However, it has been demonstrated that diagnostic performance can be increased by combining multiple significant miRNAs under the same conditions. Therefore, two, three and four combinations of miRNAs among nine candidate miRNAs were used from comparative analysis in this study and a diagnostic panel of miRNAs was developed. The performance of the diagnostic panel was verified using a validation cohort. For example, a combination of four miRNAs (miR-1246, miR-206, miR-24 and miR-373) showed AUC of 0.992 (Table V). When choosing a set of biomarker combinations, usually biomarkers having the highest AUC are selected. However, the AUC value may change as the number of samples increases. In addition, the correlation of markers and the function of each marker must be considered. A large number of biomarkers may not be an optimal set of biomarker combinations. Many factors need to be considered for commercialization. 
Table V. Performance of classification models in the training and validation sets.

A, Training set

\begin{tabular}{|c|c|c|c|c|c|c|}
\hline \multirow[b]{2}{*}{ Example of combination } & \multicolumn{6}{|c|}{ Values } \\
\hline & AUC & $\begin{array}{c}\text { Accuracy } \\
(\%)\end{array}$ & $\begin{array}{c}\text { Specificity } \\
(\%)\end{array}$ & $\begin{array}{c}\text { Sensitivity } \\
(\%)\end{array}$ & $\begin{array}{c}\text { Early stage } \\
\text { (stage 0-2) } \\
\text { Sensitivity (\%) }\end{array}$ & $\begin{array}{c}\text { Late stage } \\
\text { (stage 3-4) } \\
\text { Sensitivity (\%) }\end{array}$ \\
\hline miR-1246 & 0.955 & 88.0 & 93.0 & 85.0 & 83.0 & 95.0 \\
\hline miR-206 & 0.932 & 80.0 & 93.0 & 71.0 & 67.0 & 100.0 \\
\hline miR-24 & 0.938 & 79.0 & 93.0 & 70.0 & 66.0 & 95.0 \\
\hline miR-373 & 0.914 & 73.0 & 93.0 & 60.0 & 56.0 & 89.0 \\
\hline miR-1246+miR-206 & 0.979 & 91.0 & 93.0 & 89.0 & 87.0 & 100.0 \\
\hline miR-1246+miR-24 & 0.984 & 94.0 & 93.0 & 94.0 & 94.0 & 95.0 \\
\hline miR-1246+miR-373 & 0.968 & 94.0 & 93.0 & 94.0 & 93.0 & 100.0 \\
\hline miR-206+miR-24 & 0.976 & 90.0 & 92.0 & 88.0 & 87.0 & 95.0 \\
\hline miR-206+miR-373 & 0.964 & 87.0 & 93.0 & 83.0 & 80.0 & 100.0 \\
\hline miR-24+miR-373 & 0.982 & 92.0 & 93.0 & 92.0 & 91.0 & 95.0 \\
\hline miR-1246+miR-206+miR-24 & 1.000 & 97.0 & 93.0 & 100.0 & 100.0 & 100.0 \\
\hline miR-1246+miR-206+miR-373 & 0.985 & 94.0 & 93.0 & 95.0 & 94.0 & 100.0 \\
\hline miR-1246+miR-24+miR-373 & 0.990 & 94.0 & 93.0 & 95.0 & 95.0 & 95.0 \\
\hline miR-206+miR-24+miR-373 & 0.989 & 92.0 & 93.0 & 90.0 & 90.0 & 95.0 \\
\hline miR-1246+miR-206+miR-24+miR-373 & 0.993 & 96.0 & 93.0 & 97.0 & 97.0 & 100.0 \\
\hline
\end{tabular}

B, Validation set

Values

Example of combination

\begin{tabular}{|c|c|c|c|c|c|c|}
\hline Biomarkers & AUC & $\begin{array}{c}\text { Accuracy } \\
(\%)\end{array}$ & $\begin{array}{c}\text { Specificity } \\
(\%)\end{array}$ & $\begin{array}{c}\text { Sensitivity } \\
(\%)\end{array}$ & $\begin{array}{c}\text { (stage 0-2) } \\
\text { Sensitivity (\%) }\end{array}$ & $\begin{array}{c}\text { (stage } 3-4) \\
\text { Sensitivity }(\%)\end{array}$ \\
\hline miR-1246 & 0.963 & 90.0 & 96.0 & 86.0 & 84.0 & 100.0 \\
\hline miR-206 & 0.935 & 86.0 & 96.0 & 79.0 & 75.0 & 100.0 \\
\hline miR-24 & 0.965 & 81.0 & 96.0 & 70.0 & 65.0 & 100.0 \\
\hline miR-373 & 0.935 & 73.0 & 95.0 & 57.0 & 53.0 & 83.0 \\
\hline miR-1246+miR-206 & 0.988 & 96.0 & 98.0 & 95.0 & 94.0 & 100.0 \\
\hline miR-1246+miR-24 & 0.987 & 96.0 & 96.0 & 96.0 & 96.0 & 100.0 \\
\hline miR-1246+miR-373 & 0.983 & 93.0 & 95.0 & 92.0 & 91.0 & 100.0 \\
\hline miR-206+miR-24 & 0.973 & 91.0 & 98.0 & 86.0 & 84.0 & 100.0 \\
\hline miR-206+miR-373 & 0.981 & 91.0 & 98.0 & 86.0 & 84.0 & 100.0 \\
\hline miR-24+miR-373 & 0.977 & 96.0 & 95.0 & 96.0 & 96.0 & 100.0 \\
\hline miR-1246+miR-206+miR-24 & 0.977 & 93.0 & 86.0 & 98.0 & 97.0 & 100.0 \\
\hline miR-1246+miR-206+miR-373 & 0.991 & 96.0 & 95.0 & 96.0 & 96.0 & 100.0 \\
\hline miR-1246+miR-24+miR-373 & 0.989 & 97.0 & 96.0 & 98.0 & 97.0 & 100.0 \\
\hline miR-206+miR-24+miR-373 & 0.987 & 93.0 & 95.0 & 91.0 & 90.0 & 100.0 \\
\hline miR-1246+miR-206+miR-24+miR-373 & 0.992 & 97.0 & 96.0 & 98.0 & 97.0 & 100.0 \\
\hline
\end{tabular}

miR, microRNA; AUC, area under the curve,

Compared to a previous study (47) as an example, the combination of plasma exosome miR-1246 and miR-21 (AUC $=0.7266$ ) was a better indicator of breast cancer than individual miRNAs (AUC: 0.6914 and 0.6875, respectively).
However, the number of breast cancer patient samples used in that study (47) was $<16$ and the accuracy was lower than that of our study. Another example was a study (38) that verified the accuracy of using a combination of miR-1246, miR-1307-3p, 
miR-4634, miR-6861-5p and miR-6875-5p measured from serum for early diagnosis of breast cancer. That combination had a sensitivity of $97.3 \%$, a specificity of $82.9 \%$ and an accuracy of $89.7 \%$ for breast cancer in the test cohort. Among these five miRNAs used in that study (38), miR-1246 and miR-6875 were miRNAs that overlapped with our study. Different from our study, sera samples of Japanese breast cancer patients were used and mature miRNA expression was analysed by microarray in that study (38).

In the present study, considering that results could be influenced by storage conditions of specimens, our study used independent clinical trials from multiple institutions. Plasma samples of breast cancer patients were stored at $-80^{\circ} \mathrm{C}$ for up to 5 years. Whether such multi-miRNA set developed in this study can distinguish benign breast disease from breast cancer remains unknown. In the future, studies should be conducted using samples of patients with benign breast diseases so that benign breast diseases can be distinguished from breast cancer.

In conclusion, a set of biomarkers developed in this study showed high accuracy for early diagnosis of breast cancer. This set was prepared using a combination of two or more of nine miRNAs measured in plasma samples. It can be used to detect breast cancer at an early stage. We hope that these diagnostic indicators can be implemented to address problems of existing assistive methods for conventional mammography and effectively detect breast cancer at an early stage.

\section{Acknowledgements}

Not applicable.

\section{Funding}

Funding from BIOINFRA Life Science Inc was received.

\section{Availability of data and materials}

Datasets used and/or analysed during the current study are available from the corresponding author upon reasonable request.

\section{Authors' contributions}

JYJ and YSK conceived and designed the study, acquired data, directed drafting of the manuscript and revised it. KNK performed statistical analysis. KHK and YJP managed plasma samples and performed experiments. CWK conceived and designed the study and provided financial support. All authors read and approved the final manuscript.

\section{Ethics approval and consent to participate}

This study was ethically approved by the Institutional review board from the IRB (BIOINFRA Life Science Institutional Review Board). Plasma samples were provided by Korean Biobank through established procedures.

\section{Patient consent for publication}

Subjects whose plasma samples were used in this study provided written informed consent for the publication of their data.

\section{Competing interests}

The authors confirm that they have no competing interests.

\section{References}

1. Ferlay J, Soerjomataram I, Dikshit R, Eser S, Mathers C, Rebelo M, Parkin DM, Forman D and Bray F: Cancer incidence and mortality worldwide: Sources, methods and major patterns in GLOBOCAN 2012. Int J Cancer 136: E359-E386, 2015.

2. Wang L: Early diagnosis of breast cancer. Sensors 17: 1572, 2017.

3. Korean Breast Cancer Society: Early screening of breast cancer in Korea. J Korean Breast Cancer Soc 5: 225-234, 2002.

4. Devolli-Disha E, Manxhuka-Kërliu S, Ymeri H and Kutllovci A: Comparative accuracy of mammography and ultrasound in women with breast symptoms according to age and breast density. Bosn J Basic Med Sci 9: 131-136, 2009.

5. Marrugo-Ramírez J, Mir M and Samitier J: Blood-Based cancer biomarkers in liquid biopsy: A promising non-invasive alternative to tissue biopsy. Int J Mol Sci 19: 2877, 2018.

6. Bai Y and Zhao H: Liquid biopsy in tumors: Opportunities and challenges. Ann Transl Med 6: S89, 2018.

7. Castro-Giner F, Gkountela S, Donato C, Alborelli I, Quagliata L, $\mathrm{Ng} \mathrm{CKY}$, Piscuoglio $\mathrm{S}$ and Aceto $\mathrm{N}$ : Cancer diagnosis using a liquid biopsy: Challenges and expectations. Diagnostics (Basel) 8: 31,2018.

8. Simpson RJ, Lim JW, Moritz RL and Mathivanan S: Exosomes: Proteomic insights and diagnostic potential. Expert Rev Proteomics 6: 267-283, 2009.

9. Chen X, Liang H, Zhang J, Zen K and Zhang CY: Horizontal transfer of microRNAs: Molecular mechanisms and clinical applications. Protein Cell 3: 28-37, 2012.

10. Sanz-Rubio D, Martin-Burriel I, Gil A, Cubero P, Forner M, Khalyfa A and Marin JM: Stability of circulating Exosomal miRNAs in healthy subjects. Sci Rep 8: 10306, 2018.

11. Mitchell PS, Parkin RK, Kroh EM, Fritz BR, Wyman SK, Pogosova-Agadjanyan EL, Peterson A, Noteboom J, O'Briant KC, Allen A, et al: Circulating microRNAs as stable blood-based markers for cancer detection. Proc Natl Acad Sci USA 105: 10513-10518, 2008.

12. Salehi M and Sharifi M: Exosomal miRNAs as novel cancer biomarkers: Challenges and opportunities. J Cell Physiol 233: 6370-6380, 2018.

13. Calin GA and Croce CM: MicroRNA signatures in human cancers. Nat Rev Cancer 6: 857-866, 2006.

14. He L, He X, Lim LP, de Stanchina E, Xuan Z, Liang Y, Xue W, Zender L, Magnus J, Ridzon D, et al: A microRNA component of the p53 tumour suppressor network. Nature 447: 1130-1134, 2007.

15. Lu J, Getz G, Miska EA, Alvarez-Saavedra E, Lamb J, Peck D, Sweet-Cordero A, Ebert BL, Mak RH, Ferrando AA, et al: MicroRNA expression profiles classify human cancers. Nature 435: 834-838, 2005.

16. Calin GA, Ferracin M, Cimmino A, Di Leva G, Shimizu M, Wojcik SE, Iorio MV, Visone R, Sever NI, Fabbri M, et al: A MicroRNA signature associated with prognosis and progression in chronic lymphocytic leukemia. N Engl J Med 353: 1793-1801, 2005.

17. Schetter AJ, Leung SY, Sohn JJ, Zanetti KA, Bowman ED, Yanaihara N, Yuen ST, Chan TL, Kwong DL, Au GK, et al: MicroRNA expression profiles associated with prognosis and therapeutic outcome in colon adenocarcinoma. JAMA 299: 425-436, 2008

18. Heneghan HM, Miller N, Lowery AJ, Sweeney KJ, Newell J and Kerin MJ: Circulating microRNAs as novel minimally invasive biomarkers for breast cancer. Ann Surg 251: 499-505, 2010.

19. Zhao H, Shen J, Medico L, Wang D, Ambrosone CB and Liu S: A pilot study of circulating miRNAs as potential biomarkers of early stage breast cancer. PLoS One 5: e13735, 2010.

20. Kim VN, Han J and Siomi MC: Biogenesis of small RNAs in animals. Nat Rev Mol Cell Biol 10: 126-139, 2009.

21. Han J, Lee Y, Yeom KH, Kim YK, Jin H and Kim VN: The Drosha-DGCR8 complex in primary microRNA processing. Genes Dev 18: 3016-3027, 2004.

22. Lee Y, Ahn C, Han J, Choi H, Kim J, Yim J, Lee J, Provost P, Rådmark $\mathrm{O}, \mathrm{Kim} \mathrm{S}$ and Kim VN: The nuclear RNase III Drosha initiates microRNA processing. Nature 425: 415-419, 2003.

23. Esquela-Kerscher A and Slack FJ: Oncomirs-microRNAs with a role in cancer. Nat Rev Cancer 6: 259-269, 2006. 
24. Suzuki HI and Miyazono K: Emerging complexity of microRNA generation cascades. J Biochem 149: 15-25, 2011.

25. Salido-Guadarrama I, Romero-Cordoba S, Peralta-Zaragoza O, Hidalgo-Miranda A and Rodríguez-Dorantes M: MicroRNAs transported by exosomes in body fluids as mediators of intercellular communication in cancer. Onco Targets Ther 7: 1327-1338, 2014.

26. Lim SS and Kim YS: WO2016137031-Dumbell-structure oligonucleotide, nucleic acid amplification primer comprising same, and nucleic acid amplification method using same. KR Patent WO/2016/137031. DIOGENE Inc. Filed February 25, 2015; issued September 1, 2016.

27. Pinatel EM, Orso F, Penna E, Cimino D, Elia AR, Circosta P, Dentelli P, Brizzi MF, Provero P and Taverna D: miR-223 is a coordinator of breast cancer progression as revealed by bioinformatics predictions. PLoS One 9: e84859, 2014

28. Yoshikawa M,IinumaH,Umemoto Y, Yanagisawa T,Matsumoto A and Jinno H: Exosome-encapsulated microRNA-223-3p as a minimally invasive biomarker for the early detection of invasive breast cancer. Oncol Lett 15: 9584-9592, 2018.

29. Fu L, Li Z, Zhu J, Wang P, Fan G, Dai Y, Zheng Z and Liu Y: Serum expression levels of microRNA-382-3p, -598-3p, -1246 and -184 in breast cancer patients. Oncol Lett 12: 269-274, 2016

30. Li XJ, Ren ZJ, Tang JH and Yu Q: Exosomal MicroRNA miR-1246 Promotes Cell Proliferation, Invasion and Drug Resistance by Targeting CCNG2 in Breast Cancer. Cell Physiol Biochem 44: 1741-1748, 2017.

31. Zhou Y, Wang M, Tong Y, Liu X, Zhang L, Dong D, Shao J and Zhou Y: miR-206 promotes cancer progression by targeting Full-Length Neurokinin-1 Receptor in breast cancer. Technol Cancer Res Treat 18: 1533033819875168, 2019.

32. Khodadadi-Jamayran A, Akgol-Oksuz B, Afanasyeva Y, Heguy A, Thompson M, Ray K, Giro-Perafita A, Sánchez I, Wu X, Tripathy D, et al: Prognostic role of elevated mir-24-3p in breast cancer and its association with the metastatic process. Oncotarget 9: 12868-12878, 2018

33. Lu K, Wang J, Song Y, Zhao S, Liu H, Tang D, Pan B, Zhao H and Zhang Q: miRNA-24-3p promotes cell proliferation and inhibits apoptosis in human breast cancer by targeting p $27^{\mathrm{Kip} 1}$. Oncol Rep 34: 995-1002, 2015.

34. Eichelser C, Flesch-Janys D, Chang-Claude J, Pantel K and Schwarzenbach H: Deregulated Serum Concentrations of Circulating Cell-Free MicroRNAs miR-17, miR-34a, miR-155 and miR-373 in Human Breast Cancer Development and Progression. Clin Chem 59: 1489-1496, 2013.

35. Piasecka D, Braun M, Kordek R, Sadej R and Romanska H: MicroRNAs in regulation of triple-negative breast cancer progression. J Cancer Res Clin Oncol 144: 1401-1411, 2018.
36. Asaga S, Kuo C, Nguyen T, Terpenning M, Giuliano AE and Hoon DS: Direct serum assay for microRNA-21 concentrations in early and advanced breast cancer. Clin Chem 57: 84-91, 2011.

37. Corcoran C, Friel AM, Duffy MJ, Crown J and O'Driscoll L: Intracellular and Extracellular MicroRNAs in Breast Cancer. Clin Chem 57: 18-32, 2011.

38. Shimomura A, Shiino S, Kawauchi J, Takizawa S, Sakamoto H, Matsuzaki J, Ono M, Takeshita F, Niida S, Shimizu C, et al: Novel combination of serum microRNA for detecting breast cancer in the early stage. Cancer Sci 107: 326-334, 2016.

39. Schrauder MG, Strick R, Schulz-Wendtland R, Strissel PL, Kahmann L, Loehberg CR, Lux MP, Jud SM, Hartmann A, Hein A, et al: Circulating micro-RNAs as potential blood-based markers for early stage breast cancer detection. PLoS One 7: e29770, 2012

40. Zhao C, Li X, Han B, You Z, Qu L, Liu C, Song J, Lian L and Yang N: Gga-miR-219b targeting BCL11B suppresses proliferation, migration and invasion of Marek's disease tumor cell MSB1. Sci Rep 7: 4247, 2017.

41. Løberg M, Lousdal ML, Bretthauer M and Kalager M: Benefits and harms of mammography screening. Breast Cancer Res 17: 63, 2015.

42. Guo R, Lu G, Qin B and Fei B: Ultrasound imaging technologies for breast cancer detection and management. Ultrasound Med Biol 44: 37-70, 2018

43. Lebron-Zapata L and Jochelson MS: Overview of breast cancer screening and diagnosis. PET Clin 13: 301-323, 2018.

44. Alimirzaie S, Bagherzadeh $\mathrm{M}$ and Akbari MR: Liquid biopsy in breast cancer: A comprehensive review. Clin Genet 95: 643-660, 2019.

45. Hamam R, Hamam D, Alsaleh KA, Kassem M, Zaher W, Alfayez M, Aldahmash A and Alajez NM: Circulating microRNAs in breast cancer: Novel diagnostic and prognostic biomarkers. Cell Death Dis 8: e3045, 2017.

46. He Y, Deng F, Yang S, Wang D, Chen X, Zhong S, Zhao J and Tang J: Exosomal microRNA: A novel biomarker for breast cancer. Biomark Med 12: 177-188, 2018

47. Hannafon BN, Trigoso YD, Calloway CL, Zhao YD, Lum DH, Welm AL, Zhao ZJ, Blick KE, Dooley WC and Ding WQ: Plasma exosome microRNAs are indicative of breast cancer. Breast Cancer Res 18: 90, 2016.

This work is licensed under a Creative Commons Attribution-NonCommercial-NoDerivatives 4.0 International (CC BY-NC-ND 4.0) License. 